\title{
Bilateral Contact Problem with Adhesion between Two Bodies for Viscoelastic with Long-term Memory and Damage
}

\author{
Souida Boukrioua ${ }^{1}$, Adel Aissaoui ${ }^{2, *}$, Nacerdine Hemici $^{3}$ \\ ${ }^{1}$ Department of Mathematics, University of Ouargla, Ouargla 30000, Algeria \\ 2 Department of Mechanical Engineering, University Echahid Hamma Lakhdar El Oued, Eloued 39000, Algeria \\ ${ }^{3}$ Department of Mathematics, Ferhat Abbas Sétif University, Sétif 19000, Algeria \\ ${ }^{*}$ Corresponding Author: aissaouiadel@gmail.com
}

Copyright (C) 2017 by authors, all rights reserved. Authors agree that this article remains permanently open access under the terms of the Creative Commons Attribution License 4.0 International License

\begin{abstract}
We consider a quasistatic contact problem between two viscoelastic bodies with long-term memory and damage. The contact is bilateral and the tangential shear due to the bonding field is included. The adhesion of the contact surfaces is taken into account and modelled by a surface variable, the bonding field. We prove the existence of a unique weak solution to the problem. The proof is based on arguments of time-dependent variational inequalities, parabolic inequalities, differential equations and fixed point.
\end{abstract}

Keywords Viscoelastic Material with Long-term Memory, Damage, Adhesion, Bilateral, Weak Solution, Fixed Point

\section{Introduction}

Scientific research in mechanics are articulated around two main components: one devoted to the laws of behavior and other boundary conditions imposed on the body. The boundary conditions reflect the binding of the body with the outside world.

In this paper, we study a problem involving boundary conditions describing real phenomena such as bilateral contact and other very important such as the damage and the adhesion of two materials. For the constitutive law we consider an viscoelastic with long-term memory and damage given by

$$
\boldsymbol{\sigma}^{\ell}=\mathcal{A}^{\ell} \varepsilon\left(\dot{\mathbf{u}}^{\ell}\right)+\mathcal{G}^{\ell} \varepsilon\left(\mathbf{u}^{\ell}\right)+\int_{0}^{t} \mathcal{F}^{\ell}\left(t-s, \varepsilon\left(\mathbf{u}^{\ell}(s)\right), \alpha^{\ell}(s)\right) d s,
$$

where $\mathbf{u}^{\ell}$ the displacement field, $\boldsymbol{\sigma}^{\ell}, \alpha^{\ell}$ the damage field and $\varepsilon\left(\mathbf{u}^{\ell}\right)$ represent the stress and the linearized strain tensor, respectively. Here $\mathcal{A}^{\ell}$ is a given nonlinear operator, $\mathcal{F}^{\ell}$ is the relaxation operator, and $\mathcal{G}^{\ell}$ represents the elasticity operator. In (1) and everywhere in this paper the dot above a variable represents derivative with respect to the time variable $t$. It follows from (1) that at each time moment, the stress tensor $\boldsymbol{\sigma}^{\ell}(t)$ is split into two parts: $\boldsymbol{\sigma}^{\ell}(t)=\boldsymbol{\sigma}_{V}^{\ell}(t)+\boldsymbol{\sigma}_{R}^{\ell}(t)$, where $\sigma_{V}^{\ell}(t)=\mathcal{A}^{\ell} \varepsilon\left(\dot{\mathbf{u}}^{\ell}(t)\right)$ represents the purely viscous part of the stress, and $\sigma_{R}^{\ell}(t)$ satisfies the rate-type elastic relation

$$
\boldsymbol{\sigma}_{R}^{\ell}(t)=\mathcal{G}^{\ell} \varepsilon\left(\mathbf{u}^{\ell}(t)\right)+\int_{0}^{t} \mathcal{F}^{\ell}\left(t-s, \varepsilon\left(\mathbf{u}^{\ell}(s)\right), \alpha^{\ell}(s)\right) d s .
$$

Various results, example and mechanical interpretations in the study of elastic materials of the form (2) can be found in $[1,17]$ and references therein.

Processes of adhesion are important in many industrial settings where parts, usually nonmetallic, are glued together. For this reason, adhesive contact between deformable bodies, when a glue is added to prevent relative motion of the surfaces, has received recently increased attention in the mathematical literature. Analysis of models for adhesive contact can be found in $[2,10]$ and recently in the monographs $[11,12]$. The novelty in all these papers is the introduction of a surface internal variable, the bonding field, denoted in this paper by $\beta$. It describes the point wise fractional density of adhesion of active bonds on the contact surface, and some times it is called the intensity of adhesion. Following [6], the bonding field satisfies the restriction $0 \leq \beta \leq 1$, when $\beta=1$ at a point of the contact surface, the adhesion is complete and all the bonds are active, when $\beta=0$ all the bonds are inactive, severed, and there is no adhesion, when $0<\beta<1$ the adhesion is partial and only a fraction $\beta$ of the bonds is active. The damage is an extremely important topic in engineering, since it affects directly the useful life of the designed structure or component. There is a very large engineering literature on this topic. Models taking into account the influence of internal damage of the material on the contact process have been investigated mathematically. General models for damage were derived in [3, 4] from the virtual power principle. Mathematical analysis of one-dimensional problems can be found in [5]. The three-dimensional case has been investigated in [8]. In all these papers the damage of the material is 
described with a damage function $\alpha^{\ell}$, restricted to have values between zero and one. When $\alpha^{\ell}=1$, there is no damage in the material, when $\alpha^{\ell}=0$, the material is completely damaged, when $0<\alpha^{\ell}<1$ there is partial damage and the system has a reduced load carrying capacity. Contact problems with damage have been investigated in $[5,14,15,16]$. In this paper the inclusion used for the evolution of the damage field is

$$
\dot{\alpha}^{\ell}-\kappa^{\ell} \Delta \alpha^{\ell}+\partial \psi_{K^{\ell}}\left(\alpha^{\ell}\right) \ni \phi^{\ell}\left(\varepsilon\left(\mathbf{u}^{\ell}\right), \alpha^{\ell}\right),
$$

where $K^{\ell}$ denotes the set of admissible damage functions defined by

$$
K^{\ell}=\left\{\xi \in H^{1}\left(\Omega^{\ell}\right) ; 0 \leq \xi \leq 1, \text { a.e. in } \Omega^{\ell}\right\},
$$

$\kappa^{\ell}$ is a positive coefficient, $\partial \psi_{K^{\ell}}$ represents the subdifferential of the indicator function of the set $K^{\ell}$ and $\phi^{\ell}$ is a given constitutive function which describes the sources of the damage in the system. In this article we consider a mathematical frictional contact problem between two viscoelastic bodies with constitutive law with long-term memory and damage. The contact is modelled with normal compliance where the adhesion of the contact surfaces is taken into account and is modelled with a surface variable, the bonding field. We derive a variational formulation of the problem and prove the existence of a unique weak solution.

This article is organized as follows. In Section 2 we describe the mathematical models for the frictional contact problem between two viscoelastic bodies with long-term memory and damage. The contact is modelled with normal compliance and adhesion. In Section 3 we introduce some notation, list the assumptions on the problem's data, and derive the variational formulation of the model. In section 4 we present our main result stated in Theorem 4.1 and its proof which is based on arguments of time-dependent variational inequalities, parabolic inequalities, differential equations and fixed point.

\section{Problem Statement}

We describe the model for the process, we present its variational formulation. The physical setting is as follows. Let us consider two viscoelastic bodies with long-term memory occupying two bounded domains $\Omega^{1}, \Omega^{2}$ of the space $\mathbb{R}^{d}(d=2,3)$. For each domain $\Omega^{\ell}$, the boundary $\Gamma^{\ell}$ is assumed to be Lipschitz continuous, and is partitioned into three disjoint measurable parts $\Gamma_{1}^{\ell}, \Gamma_{2}^{\ell}$ and $\Gamma_{3}^{\ell}$, on one hand, such that meas $\Gamma_{1}^{\ell}>0$. Let $T>0$ and let $[0, T]$ be the time interval of interest. The body $\Omega^{\ell}$ is subjected to $\boldsymbol{f}_{0}^{\ell}$ forces. The bodies are assumed to be clamped on $\Gamma_{1}^{\ell} \times(0, T)$. The surface tractions $\boldsymbol{f}_{2}^{\ell}$ act on $\Gamma_{2}^{\ell} \times(0, T)$. The two bodies can enter in contact along the common part $\Gamma_{3}^{1}=\Gamma_{3}^{2}=\Gamma_{3}$. The bodies are in adhesive contact with an obstacle, over the contact surface $\Gamma_{3}$. With the assumption above, the classical formulation of the contact problem with adhesion and damage between two viscoelastics bodies with long-term memory is following.

\section{Problem P}

Find the displacement field $\mathbf{u}=\left(\mathbf{u}^{1}, \mathbf{u}^{2}\right)$ such that $\mathbf{u}^{\ell}$ : $\Omega^{\ell} \times[0, T] \rightarrow \mathbb{R}^{d}$, a stress field $\boldsymbol{\sigma}=\left(\boldsymbol{\sigma}^{1}, \boldsymbol{\sigma}^{2}\right)$ such that $\boldsymbol{\sigma}^{\ell}$ : $\Omega^{\ell} \times[0, T] \rightarrow \mathbb{S}^{d}$, a damage field $\alpha=\left(\alpha^{1}, \alpha^{2}\right)$ such that $\alpha^{\ell}: \Omega^{\ell} \times[0, T] \rightarrow \mathbb{R}$ and a bonding field $\beta: \Gamma_{3} \times[0, T] \rightarrow \mathbb{R}$ such that

$$
\begin{aligned}
& \sigma^{\ell}=\mathcal{A}^{\ell} \varepsilon\left(\dot{\mathbf{u}}^{\ell}\right)+\mathcal{G}^{\ell}\left(\varepsilon\left(\mathbf{u}^{\ell}\right)\right) \\
& +\int_{0}^{t} \mathcal{F}^{\ell}\left(t-s, \varepsilon\left(\mathbf{u}^{\ell}(s)\right), \alpha^{\ell}(s)\right) d s \\
& \dot{\alpha}^{\ell}-k^{\ell} \Delta \alpha^{\ell}+\partial \varphi_{K^{\ell}}\left(\alpha^{\ell}\right) \ni \phi^{\ell}\left(\varepsilon\left(\mathbf{u}^{\ell}\right), \alpha^{\ell}\right), \text { in } \Omega^{\ell} \times(0, T),
\end{aligned}
$$

$$
\begin{aligned}
& \text { Div } \boldsymbol{\sigma}^{\ell}+f_{0}^{\ell}=\mathbf{0}, \text { in } \Omega^{\ell} \times(0, T), \\
& \mathbf{u}^{\ell}=\mathbf{0}, \text { on } \Gamma_{1}^{\ell} \times(0, T), \\
& \boldsymbol{\sigma}^{\ell} \boldsymbol{\nu}^{\ell}=f_{2}^{\ell}, \text { on } \Gamma_{2}^{\ell} \times(0, T), \\
& \boldsymbol{\sigma}_{\nu}^{1}=\boldsymbol{\sigma}_{\nu}^{2}, \quad \mathbf{u}_{\nu}^{1}+\mathbf{u}_{\nu}^{2}=0, \text { on } \Gamma_{3} \times(0, T), \\
& -\sigma_{\tau}^{1}=\sigma_{\tau}^{2}=p_{\tau}\left(\beta, u_{\tau}^{1}-u_{\tau}^{2}\right), \text { on } \Gamma_{3} \times(0, T), \\
& \dot{\beta}=H_{a d}\left(\beta, \zeta_{\beta}, R\left(\left|u_{\tau}^{1}-u_{\tau}^{2}\right|\right)\right), \text { on } \Gamma_{3} \times(0, T), \\
& \frac{\partial \alpha^{\ell}}{\partial \nu^{\ell}}=0, \text { on } \Gamma^{\ell} \times(0, T), \\
& \mathbf{u}^{\ell}(0)=\mathbf{u}_{0}^{\ell} \quad \alpha^{\ell}(0)=\alpha_{0}^{\ell}, \text { in } \Omega^{\ell}, \\
& \beta(0)=\beta_{0}, \text { on } \Gamma_{3} .
\end{aligned}
$$

First, equations (5) represent the viscoelastic constitutive law with long term-memory and damage, the evolution of the damage is governed by the inclusion of parabolic type given by the relation (6) where $\phi$ is the mechanical source of the damage growth, assumed to be a rad her general function of the strains and damage itself, and $\partial \varphi_{K}$ is the subdifferential of the indicator function of the admissible damage functions set $K$. Equations (7) are the equilibrium equations for the stress, in which "Div" denote the divergence operator for tensor. (8) and (9) are the displacement and traction boundary conditions, respectively. Condition (10) shows that the contact is bilateral, i.e. there is no separation between the bodies during the process, while condition (11) shows that the resistance to tangential motion is generated by the glue, in comparison to which the frictional traction can be neglected. Moreover, the tangential traction depends only on the bonding field and on the relative tangential displacement. Next, equation (12) governs the evolution of the adhesion field is assumed to depend generally on $\beta$ and $|\mathbf{u}|$, can be found in $[19,21,22,23]$. We do not impose sign restrictions on the process, and thus, cycles of debonding and rebonding may take place, as a result of imposed periodic forces. In addition, we include here the possibility that, as the cycle of bonding and debonding go on, there is a decrease in the bonding effectiveness. Therefore, the process is also assumed to depend on the time history of the bonding, which we denote by

$$
\zeta_{\beta}(\mathbf{x}, t)=\int_{0}^{t} \beta(\mathbf{x}, s) d s \quad \text { on } \Gamma_{3} \times(0, T) .
$$

The whole process is assumed to be governed by the differential equation

$$
\dot{\beta}=H_{a d}\left(\beta, \zeta_{\beta}, R(|\mathbf{u}|)\right) \quad \text { on } \Gamma_{3} \times(0, T) .
$$


Here, $H_{a d}$ is a general function discussed below, which vanishes when its first argument vanishes.

An example of such that a function is

$$
H_{a d}(\beta, r)=-\gamma_{\nu} \beta_{+} r^{2},
$$

where $\gamma_{\nu}$ is the bonding energy constant and $\beta_{+}$is the maximal tensile normal traction that the adhesive can provide. We note that in this case only debonding is allowed. Another example, in which $H_{a d}$ depends on all three variables is

$$
H_{a d}\left(\beta, \zeta_{\beta}, r\right)=-\gamma_{\nu} \beta_{+} r^{2}+\frac{\gamma_{\nu} \beta_{+}(1-\beta)_{+}}{1+\zeta_{\beta}^{2}} .
$$

Here the magnitude of the displacement $r$ causes the debonding, while naturally there is a tendency to rebond. However, the bonding cannot exceed $\beta=1$, and, moreover, the rebonding becomes weaker as the process goes on, which is represented by the factor $1+\zeta_{\beta}^{2}$ in the denominator. Equation (13) represents a homogeneous Newmann boundary condition where $\frac{\partial \alpha}{\partial \nu}$ represents the normal derivative of $\alpha$. In (14) we consider the initial conditions where $\mathbf{u}_{0}$ is the initial displacement, and $\alpha_{0}$ the initial damage. Finally, (15) is the initial condition, in which $\beta_{0}$ denotes the initial bonding.

\section{Variational formulation and the main result}

In this section, we list the assumptions on the data and derive a variational formulation for the contact problem. To this end, we need to introduce some notation and preliminary material. Here and below, $\mathbb{S}^{d}$ represent the space of secondorder symmetric tensors on $\mathbb{R}^{d}$. We recall that the inner products and the corresponding norms on $\mathbb{S}^{d}$ and $\mathbb{R}^{d}$ are given by

$$
\begin{aligned}
& \mathbf{u}^{\ell} \cdot \mathbf{v}^{\ell}=u_{i}^{\ell} \cdot v_{i}^{\ell}, \quad\left|\mathbf{v}^{\ell}\right|=\left(\mathbf{v}^{\ell} \cdot \mathbf{v}^{\ell}\right)^{\frac{1}{2}}, \quad \forall \mathbf{u}^{\ell}, \mathbf{v}^{\ell} \in \mathbb{R}^{d}, \\
& \boldsymbol{\sigma}^{\ell} \cdot \boldsymbol{\tau}^{\ell}=\sigma_{i j}^{\ell} \cdot \tau_{i j}^{\ell}, \quad\left|\boldsymbol{\tau}^{\ell}\right|=\left(\boldsymbol{\tau}^{\ell} \cdot \boldsymbol{\tau}^{\ell}\right)^{\frac{1}{2}}, \quad \forall \boldsymbol{\sigma}^{\ell}, \boldsymbol{\tau}^{\ell} \in \mathbb{S}^{d} .
\end{aligned}
$$

Here and below, the indices $i$ and $j$ run between 1 and $d$ and the summation convention over repeated indices is adopted. Now, to proceed with the variational formulation, we need the following function spaces:

$$
\begin{aligned}
& H^{\ell}=\left\{\mathbf{v}^{\ell}=\left(v_{i}^{\ell}\right) ; v_{i}^{\ell} \in L^{2}\left(\Omega^{\ell}\right)\right\}, \\
& H_{1}^{\ell}=\left\{\mathbf{v}^{\ell}=\left(v_{i}^{\ell}\right) ; v_{i}^{\ell} \in H^{1}\left(\Omega^{\ell}\right)\right\}, \\
& \mathcal{H}^{\ell}=\left\{\boldsymbol{\tau}^{\ell}=\left(\tau_{i j}^{\ell}\right) ; \tau_{i j}^{\ell}=\tau_{j i}^{\ell} \in L^{2}\left(\Omega^{\ell}\right)\right\}, \\
& \mathcal{H}_{1}^{\ell}=\left\{\boldsymbol{\tau}^{\ell}=\left(\tau_{i j}^{\ell}\right) \in \mathcal{H}^{\ell} ; \operatorname{div} \boldsymbol{\tau}^{\ell} \in H^{\ell}\right\} .
\end{aligned}
$$

The spaces $H^{\ell}, H_{1}^{\ell}, \mathcal{H}^{\ell}$ and $\mathcal{H}_{1}^{\ell}$ are real Hilbert spaces endowed with the canonical inner products given by

$$
\begin{aligned}
& \left(\mathbf{u}^{\ell}, \mathbf{v}^{\ell}\right)_{H^{\ell}}=\int_{\Omega^{\ell}} \mathbf{u}^{\ell} \cdot \mathbf{v}^{\ell} d x, \\
& \left(\mathbf{u}^{\ell}, \mathbf{v}^{\ell}\right)_{H_{1}^{\ell}}=\int_{\Omega^{\ell}} \mathbf{u}^{\ell} \cdot \mathbf{v}^{\ell} d x+\int_{\Omega^{\ell}} \nabla \mathbf{u}^{\ell} \cdot \nabla \mathbf{v}^{\ell} d x,
\end{aligned}
$$

$$
\begin{aligned}
& \left(\boldsymbol{\sigma}^{\ell}, \boldsymbol{\tau}^{\ell}\right)_{\mathcal{H}^{\ell}}=\int_{\Omega^{\ell}} \boldsymbol{\sigma}^{\ell} \cdot \boldsymbol{\tau}^{\ell} d x, \\
& \left(\boldsymbol{\sigma}^{\ell}, \boldsymbol{\tau}^{\ell}\right)_{\mathcal{H}_{1}^{\ell}}=\int_{\Omega^{\ell}} \boldsymbol{\sigma}^{\ell} \cdot \boldsymbol{\tau}^{\ell} d x+\int_{\Omega^{\ell}} \operatorname{div} \boldsymbol{\sigma}^{\ell} . \operatorname{Div} \boldsymbol{\tau}^{\ell} d x
\end{aligned}
$$

and the associated norms $\|\cdot\|_{H^{\ell}},\|\cdot\|_{H_{1}^{\ell}},\|\cdot\|_{\mathcal{H}^{\ell}}$, and $\|\cdot\|_{\mathcal{H}_{1}^{\ell}}$ respectively. Here and below we use the notation

$$
\begin{gathered}
\nabla \mathbf{u}^{\ell}=\left(u_{i, j}^{\ell}\right), \quad \varepsilon\left(\mathbf{u}^{\ell}\right)=\left(\varepsilon_{i j}\left(\mathbf{u}^{\ell}\right)\right), \\
\varepsilon_{i j}\left(\mathbf{u}^{\ell}\right)=\frac{1}{2}\left(u_{i, j}^{\ell}+u_{j, i}^{\ell}\right), \quad \forall u^{\ell} \in H_{1}^{\ell}, \\
\operatorname{Div} \boldsymbol{\sigma}^{\ell}=\left(\sigma_{i j, j}^{\ell}\right), \quad \forall \boldsymbol{\sigma}^{\ell} \in \mathcal{H}_{1}^{\ell} .
\end{gathered}
$$

When $\sigma^{\ell}$ is a regular function, the following Green's type formula holds,

$$
\left(\boldsymbol{\sigma}^{\ell} \boldsymbol{\nu}^{\ell}, \mathbf{v}^{\ell}\right)_{\Gamma^{\ell}}=\left(\boldsymbol{\sigma}^{\ell}, \varepsilon\left(\mathbf{v}^{\ell}\right)\right)_{\mathcal{H}^{\ell}}+\left(\operatorname{Div} \boldsymbol{\sigma}^{\ell}, \mathbf{v}^{\ell}\right)_{H^{\ell}} \quad \forall \mathbf{v}^{\ell} \in H_{1}^{\ell} .
$$

To obtain the variational formulation of the problem (5)(15), we introduce for the bonding field the set

$$
\begin{array}{r}
\mathcal{Z}=\left\{\theta \in L^{\infty}\left(0, T ; L^{2}\left(\Gamma_{3}\right)\right) ; 0 \leq \theta(t) \leq 1,\right. \\
\left.\forall t \in[0, T], \text { a.e. on } \Gamma_{3}\right\},
\end{array}
$$

and for the displacement field we need the closed subspace of $H_{1}^{\ell}$ defined by

$$
V^{\ell}=\left\{\mathbf{v}^{\ell} \in H_{1}^{\ell} ; \mathbf{v}^{\ell}=0 \text { on } \Gamma_{1}^{\ell}\right\} .
$$

Since meas $\Gamma_{1}^{\ell}>0$, the following Korn's inequality holds:

$$
\left\|\varepsilon\left(\mathbf{v}^{\ell}\right)\right\|_{\mathcal{H}^{\ell}} \geq c_{K}\left\|\mathbf{v}^{\ell}\right\|_{H_{1}^{\ell}} \quad \forall \mathbf{v}^{\ell} \in V^{\ell},
$$

where the constant $c_{K}$ denotes a positive constant which may depends only on $\Omega^{\ell}, \Gamma_{1}^{\ell}$ (see [11]). Over the space $V^{\ell}$ we consider the inner product given by

$$
\left(\mathbf{u}^{\ell}, \mathbf{v}^{\ell}\right)_{V^{\ell}}=\left(\varepsilon\left(\mathbf{u}^{\ell}\right), \varepsilon\left(\mathbf{v}^{\ell}\right)\right)_{\mathcal{H}^{\ell}}, \quad \forall \mathbf{u}^{\ell}, \mathbf{v}^{\ell} \in V^{\ell},
$$

and let $\|\cdot\|_{V^{e}}$ be the associated norm. It follows from Korn's inequality (20) that the norms $\|\cdot\|_{H_{1}^{\ell}}$ and $\|\cdot\|_{V^{\ell}}$ are equivalent on $V^{\ell}$. Then $\left(V^{\ell},\|\cdot\|_{V^{\ell}}\right)$ is a real Hilbert space. Moreover, by the Sobolev trace theorem and (21), there exists a constant $c_{0}>0$, depending only on $\Omega^{\ell}, \Gamma_{1}^{\ell}$ and $\Gamma_{3}$ such that

$$
\left\|\mathbf{v}^{\ell}\right\|_{L^{2}\left(\Gamma_{3}\right)^{d}} \leq c_{0}^{\ell}\left\|\mathbf{v}^{\ell}\right\|_{V^{\ell}} \quad \forall \mathbf{v}^{\ell} \in V^{\ell} .
$$

We define the set $\boldsymbol{V}$ of admissible displacement fields by

$$
\boldsymbol{V}=\left\{\mathbf{v}=\left(\mathbf{v}^{1}, \mathbf{v}^{2}\right) \in V^{1} \times V^{2} \mid v_{\nu}^{1}+v_{\nu}^{2}=0 \text { on } \Gamma_{3}\right\},
$$

We also introduce the spaces $E_{0}^{\ell}=L^{2}\left(\Omega^{\ell}\right), E_{1}^{\ell}=H^{1}\left(\Omega^{\ell}\right)$. To simplify notation, we define the product spaces

$$
\begin{aligned}
\mathcal{H}=\mathcal{H}^{1} \times \mathcal{H}^{2}, & \mathcal{H}_{1}=\mathcal{H}_{1}^{1} \times \mathcal{H}_{1}^{2}, \\
E_{0}=E_{0}^{1} \times E_{0}^{2}, & E_{1}=E_{1}^{1} \times E_{1}^{2},
\end{aligned}
$$

The spaces $\boldsymbol{V}$ and $E_{1}$, and are real Hilbert spaces endowed with the canonical inner products denoted by $(\cdot, \cdot)_{\boldsymbol{V}}$ and $(\cdot, \cdot)_{E_{1}}$. The associate norms will be denoted by $\|\cdot\|_{V}$ and $\|\cdot\|_{E_{1}}$ respectively. 
Finally, for any real Hilbert space $X$, we use the classical notation for the spaces $L^{p}(0, T ; X), W^{k, p}(0, T ; X)$, where $1 \leq$ $p \leq \infty, k \geq 1$. We denote by $C(0, T ; X)$ and $C^{1}(0, T ; X)$ the space of continuous and continuously differentiable functions from $[0, T]$ to $X$, respectively, with the norms

$$
\begin{gathered}
\|f\|_{C(0, T ; X)}=\max _{t \in[0, T]}\|f(t)\|_{X}, \\
\|f\|_{C^{1}(0, T ; X)}=\max _{t \in[0, T]}\|f(t)\|_{X}+\max _{t \in[0, T]}\|\dot{f}(t)\|_{X},
\end{gathered}
$$

respectively. Moreover, we use the dot above to indicate the derivative with respect to the time variable and, for areal number $r$, we use $r_{+}$to represent its positive part, that is $r_{+}=\max \{0, r\}$. For the convenience of the reader, we recall the following version of the classical theorem of CauchyLipschitz (see, [16, p.48]).

Theorem 3.1 Assume that $\left(X,\|\cdot\|_{X}\right)$ is a real Banach space and $T>0$. Let $F(t, \cdot): X \rightarrow X$ be an operator defined a.e. on $(0, T)$ satisfying the following conditions:

There exists a constant $L_{F}>0$ such that

$$
\begin{gathered}
\|F(t, x)-F(t, y)\|_{X} \leq L_{F}\|x-y\|_{X} \\
\forall x, y \in X, \text { a.a. } t \in(0, T) .
\end{gathered}
$$

There exists $p \geq 1$ such that $t \mapsto F(t, x) \in L^{p}(0, T ; X)$ for all $x \in X$.

Then for any $x_{0} \in X$, there exists a unique function $x \in$ $W^{1, p}(0, T ; X)$ such that

$$
\begin{gathered}
\dot{x}(t)=F(t, x(t)), \quad \text { a.e. } t \in(0, T), \\
x(0)=x_{0} .
\end{gathered}
$$

This theorem 4.1 will be used in section 4 to prove the unique solvability of the intermediate problem involving the bonding field.

In the study of the Problem P, we consider the following assumptions:

The viscosity function $\mathcal{A}^{\ell}: \Omega^{\ell} \times \mathbb{S}^{d} \rightarrow \mathbb{S}^{d}$ satisfies:
(a) There exists $L_{\mathcal{A}^{\ell}}>0$ such that $\left|\mathcal{A}^{\ell}\left(x, \xi_{1}\right)-\mathcal{A}^{\ell}\left(x, \xi_{2}\right)\right| \leq L_{\mathcal{A}^{\ell}}\left|\xi_{1}-\xi_{2}\right|$, $\forall \xi_{1}, \xi_{2} \in \mathbb{S}^{d}$, a.e. $x \in \Omega^{\ell}$,
(b) There exists $m_{\mathcal{A}^{\ell}}>0$ such that $\left(\mathcal{A}^{\ell}\left(x, \xi_{1}\right)-\mathcal{A}^{\ell}\left(x, \xi_{2}\right)\right) \cdot\left(\xi_{1}-\xi_{2}\right) \geq m_{\mathcal{A}^{\ell}}\left|\xi_{1}-\xi_{2}\right|^{2}$ $\forall \xi_{1}, \xi_{2} \in \mathbb{S}^{d}$, a.e. $x \in \Omega^{\ell}$,
(c) The map $x \mapsto \mathcal{A}^{\ell}(x, \xi)$ is Lebesgue measurable on $\Omega^{\ell}$ for any $\xi \in \mathbb{S}^{d}$,
(d) The map $x \mapsto \mathcal{A}^{\ell}(x, 0) \in \mathcal{H}^{\ell}$.

The elasticity operator $\mathcal{G}^{\ell}: \Omega^{\ell} \times \mathbb{S}^{d} \rightarrow \mathbb{S}^{d}$ satisfies:

$$
\left\{\begin{array}{l}
\text { (a) There exists } L_{\mathcal{G}^{\ell}}>0 \text { such that } \\
\left|\mathcal{G}^{\ell}\left(x, \xi_{1}\right)-\mathcal{G}^{\ell}\left(x, \xi_{2}\right)\right| \leq L_{\mathcal{G}^{\ell}}\left|\xi_{1}-\xi_{2}\right|, \\
\forall \xi_{1}, \xi_{2} \in \mathbb{S}^{d} \text {, a.e. } x \in \Omega^{\ell}, \\
\text { (b) For any } \xi \in \mathbb{S}^{d} \text { and }, \\
x \mapsto \mathcal{G}^{\ell}(x, \xi) \text { is Lebesgue measurable on } \Omega^{\ell}, \\
\text { (c) The map } x \mapsto \mathcal{G}^{\ell}(x, 0) \in \mathcal{H}^{\ell} .
\end{array}\right.
$$

The relaxation function $\mathcal{F}^{\ell}: \Omega^{\ell} \times(0, T) \times \mathbb{S}^{d} \times \mathbb{R} \rightarrow \mathbb{S}^{d}$ satisfies:

$$
\begin{aligned}
& \text { (a) There exists } \mathcal{F}^{\ell}>0 \text { such that } \\
& \left|\mathcal{F}^{\ell}\left(\boldsymbol{x}, t, \boldsymbol{\xi}_{1}, d_{1}\right)-\mathcal{F}^{\ell}\left(\boldsymbol{x}, t, \boldsymbol{\xi}_{2}, d_{2}\right)\right| \\
& \leq L_{\mathcal{F} \ell}\left(\left|\boldsymbol{\xi}_{1}-\boldsymbol{\xi}_{2}\right|+\left|d_{1}-d_{2}\right|\right)
\end{aligned}
$$

for all $t \in(0, T), \boldsymbol{\xi}_{1}, \boldsymbol{\xi}_{2} \in \mathbb{S}^{d}$,

$d_{1}, d_{2} \in \mathbb{R}$, a.e. $\boldsymbol{x} \in \Omega^{\ell}$.
(b) The mapping $\boldsymbol{x} \mapsto \mathcal{F}^{\ell}(\boldsymbol{x}, t, \boldsymbol{\xi}, d)$ is Lebesgue
measurable in $\Omega^{\ell}$, for any $t \in(0, T), \boldsymbol{\xi} \in \mathbb{S}^{d}, d \in \mathbb{R}$.
(c) The mapping $t \mapsto \mathcal{F}^{\ell}(\boldsymbol{x}, t, \boldsymbol{\xi}, d)$ is continuous in ,
$(0, T)$ for any $\boldsymbol{\xi} \in \mathbb{S}^{d}, d \in \mathbb{R}$, a.e. $\boldsymbol{x} \in \Omega^{\ell}$.
(d) The mapping $\boldsymbol{x} \mapsto \mathcal{F}^{\ell}(\boldsymbol{x}, t, \mathbf{0}, 0)$ belong,
to $\mathcal{H}^{\ell}$ for all $t \in(0, T)$.

The damage source function $\phi^{\ell}: \Omega^{\ell} \times \mathbb{S}^{d} \times \times \mathbb{R} \rightarrow \mathbb{R}$ satisfies:

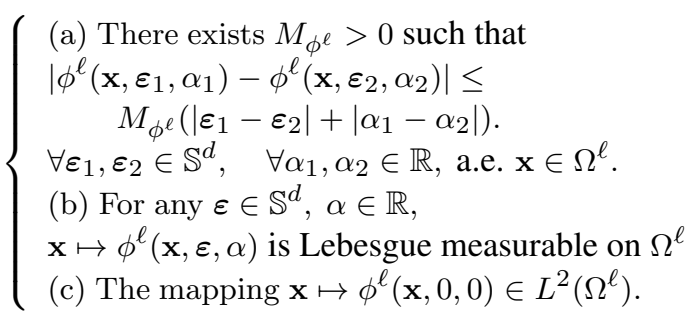

The tangential contact function $p_{\tau}: \Gamma_{3} \times \mathbb{R} \times \mathbb{R}^{d} \rightarrow \mathbb{R}^{d}$ satisfies:
(a) There exists $L_{\tau}>0$ such that $\left|p_{\tau}\left(x, \beta_{1}, r_{1}\right)-p_{\tau}\left(x, \beta_{2}, r_{2}\right)\right| \leq L_{\tau}\left(\left|\beta_{1}-\beta_{2}\right|+\left|r_{1}-r_{2}\right|\right)$
$\forall \beta_{1}, \beta_{2} \in \mathbb{R}, r_{1}, r_{2} \in \mathbb{R}^{d}$, a.e. $x \in \Gamma_{3} ;$
(b) The map $x \mapsto p_{\tau}(x, \beta, r)$ is Lebesgue measurable on $\Gamma_{3}$ $\forall \beta \in \mathbb{R}, r \in \mathbb{R}^{d} ;$
(c) The map $x \mapsto p_{\tau}(x, 0,0) \in L^{\infty}\left(\Gamma_{3}\right)^{d}$;
(d) $p_{\tau}(x, \beta, r) . \nu(x)=0 \quad \forall r \in \mathbb{R}^{d}$ such that $r . \nu(x)=0$, a.e. $x \in \Gamma_{3}$.

The adhesion function $H_{a d}: \Gamma_{3} \times \mathbb{R} \times[-L, L] \rightarrow \mathbb{R}$ satisfies:

(a) There exists $L_{H_{a d}}>0$ such that

$$
\begin{aligned}
& \left|H_{a d}\left(x, b_{1}, r_{1}\right)-H_{a d}\left(x, b_{2}, r_{2}\right)\right| \leq \\
& L_{H a d}\left(\left|b_{1}-b_{2}\right|+\left|r_{1}-r_{2}\right|\right) \\
& \forall b_{1}, b_{2} \in \mathbb{R} \forall r_{1}, r_{2} \in[-L, L] \text { a.e. } x \in \Gamma_{3},
\end{aligned}
$$

(b) The map $x \rightarrow H_{a d}(x, b, r)$ is Lebesgue measurable on $\Gamma_{3}$ $\forall b \in \mathbb{R} r \in[-L, L]$,

(c) The map $(b, r) \mapsto H_{a d}(x, b, r)$ is continuous

on $\mathbb{R} \times[-L, L]$ a.e. $x \in \Gamma_{3}$,
(d) $H_{a d}(x, 0, r)=0 \quad \forall r \in[-L, L]$, a.e. $x \in \Gamma_{3}$,
(e) $H_{a d}(x, b, r) \geq 0 \quad \forall b \leq 0, r \in[-L, L]$, a.e. $x \in \Gamma_{3}$ and $H_{a d}(x, b, r) \leq 0 \quad \forall b \geq 1, r \in[-L, L]$, a.e. $x \in \Gamma_{3}$.

We also suppose that the body forces and surface tractions satisfy

$$
\mathbf{f}_{0}^{\ell} \in C\left(0, T ; H^{\ell}\right), \quad \mathbf{f}_{2}^{\ell} \in C\left(0, T ; L^{2}\left(\Gamma_{2}^{\ell}\right)^{d}\right),
$$

and, finally, the initial data satisfies

$$
\beta_{0} \in L^{\infty}\left(\Gamma_{3}\right), \quad 0 \leq \beta_{0} \leq 1 \text { a.e. } x \in \Gamma_{3} .
$$


Finally we assume that the initial data satisfy the following conditions

$$
\begin{aligned}
& \mathbf{u}_{0}^{\ell} \in V^{\ell}, \\
& \alpha_{0}^{\ell} \in K^{\ell} .
\end{aligned}
$$

We define the bilinear forme $a: H^{1}\left(\Omega^{\ell}\right) \times H^{1}\left(\Omega^{\ell}\right) \rightarrow \mathbb{R}$ by

$$
a(\zeta, \varphi)=\sum_{\ell=1}^{2} k^{\ell} \int_{\Omega^{\ell}} \nabla \zeta^{\ell} \cdot \nabla \varphi^{\ell} d x
$$

The microcrack diffusion coefficient verifies

$$
k^{\ell}>0
$$

We define the map $\mathbf{f}=\left(\mathbf{f}^{1}, \mathbf{f}^{2}\right):[0, T] \rightarrow V$ by the equality

$$
(\mathbf{f}(t), \mathbf{v})_{V}=\sum_{\ell=1}^{2}\left(\left(\mathbf{f}_{0}^{\ell}(t), \mathbf{v}^{\ell}\right)_{H^{\ell}}+\int_{\Gamma_{2}^{\ell}} \mathbf{f}_{2}^{\ell}(t) \cdot \mathbf{v}^{\ell} d a\right)
$$

$\forall \mathbf{v}=\left(\mathbf{v}^{1}, \mathbf{v}^{2}\right) \in V$, a.e.t $\in(0 ; T)$. We note that, using (29) we obtain the following regularity

$$
\mathbf{f} \in C(0, T ; V) \text {. }
$$

Using on formula Green's and (7), we deduce that for $\ell=$ 1,2 we have

$$
\begin{aligned}
& \left(\boldsymbol{\sigma}^{\ell}(t), \boldsymbol{\varepsilon}\left(\mathbf{v}^{\ell}\right)\right)_{\mathcal{H}^{\ell}}=\left(\mathbf{f}_{0}^{\ell}(t), \mathbf{v}^{\ell}\right)_{H^{\ell}}+ \\
& \int_{\Gamma_{2}^{\ell}} \mathbf{f}_{2}^{\ell}(t) \cdot \mathbf{v}^{\ell} d a+\int_{\Gamma_{3}}\left(\sigma_{\nu}^{\ell}(t) v_{\nu}^{\ell}+\boldsymbol{\sigma}_{\tau}^{\ell}(t) \cdot \mathbf{v}_{\tau}^{\ell}\right) d a, \\
& \forall \mathbf{v}^{\ell} \in V^{\ell} \quad \text { a.e. } t \in(0 ; T) .
\end{aligned}
$$

From (35) and (37) we deduce

$$
\begin{aligned}
& \sum_{\ell=1}^{2}\left(\boldsymbol{\sigma}^{\ell}(t), \boldsymbol{\varepsilon}\left(\mathbf{v}^{\ell}\right)\right)_{\mathcal{H}^{\ell}}=(\mathbf{f}(t), \mathbf{v})_{V}+ \\
& \sum_{\ell=1}^{2} \int_{\Gamma_{3}}\left(\sigma_{\nu}^{\ell}(t) v_{\nu}^{\ell}+\boldsymbol{\sigma}_{\tau}^{\ell}(t) \cdot \mathbf{v}_{\tau}^{\ell}\right) d a
\end{aligned}
$$

$\forall \mathbf{v}^{\ell} \in V^{\ell}$ a.e. $t \in(0 ; T)$. Keeping in mind (10) and (11) we deduce

$\sum_{\ell=1}^{2} \int_{\Gamma_{3}}\left(\sigma_{\nu}^{\ell} v_{\nu}^{\ell}+\boldsymbol{\sigma}_{\tau}^{\ell} \cdot \mathbf{v}_{\tau}^{\ell}\right) d a=-\int_{\Gamma_{3}} p_{\tau}\left(\beta, \mathbf{u}_{\tau}^{1}-\mathbf{u}_{\tau}^{2}\right) \cdot\left(\mathbf{v}_{\tau}^{1}-\mathbf{v}_{\tau}^{2}\right) d a$

Let define the functional $j: L^{\infty}\left(\Gamma_{3}\right) \times V \times V \rightarrow \mathbb{R}$ by

$$
j(\beta, \mathbf{u}, \mathbf{v})=\int_{\Gamma_{3}} \mathbf{p}_{\tau}\left(\beta, \mathbf{u}_{\tau}^{1}-\mathbf{u}_{\tau}^{2}\right) \cdot\left(\mathbf{v}_{\tau}^{1}-\mathbf{v}_{\tau}^{2}\right) d a,
$$

$\forall \beta \in L^{\infty}\left(\Gamma_{3}\right), \forall \mathbf{u}=\left(\mathbf{u}^{1}, \mathbf{u}^{2}\right) \in V$ and $\mathbf{v}=\left(\mathbf{v}^{1}, \mathbf{v}^{2}\right) \in V$.

By a standard procedure based on Green's formula, we derive the following variational formulation of the mechanical (5)-(15).

\section{Problem PV}

Find a displacement field $\mathbf{u}=\left(\mathbf{u}^{1}, \mathbf{u}^{2}\right):[0, T] \rightarrow \boldsymbol{V}$, a a damage field $\alpha=\left(\alpha^{1}, \alpha^{2}\right):[0, T] \rightarrow E_{1}$, and a bonding field $\beta:[0, T] \rightarrow L^{\infty}\left(\Gamma_{3}\right)$ such that

$$
\begin{aligned}
& \sum_{\ell=1}^{2}\left(\mathcal{A}^{\ell} \varepsilon\left(\dot{\mathbf{u}}^{\ell}(t)\right), \varepsilon(v)\right)_{\mathcal{H}}+ \\
& \sum_{\ell=1}^{2}\left(\int_{0}^{t} \mathcal{F}^{\ell}\left(t-s, \varepsilon\left(\mathbf{u}^{\ell}(s)\right), \alpha^{\ell}(s)\right) d s, \varepsilon(v)\right)_{\mathcal{H}^{+}} \\
& \sum_{\ell=1}^{2}\left(\mathcal{G}^{\ell} \varepsilon\left(\mathbf{u}^{\ell}(t)\right), \varepsilon(v)\right)_{\mathcal{H}}+j(\beta(t), \mathbf{u}(t), \mathbf{v})=(\mathbf{f}(t), \mathbf{v})_{V}, \\
& \quad \forall \mathbf{v} \in V, \\
& \quad \geq \sum_{\ell=1}^{2}\left(\phi^{\ell}\left(\varepsilon\left(\mathbf{u}^{\ell}(t)\right), \alpha^{\ell}(t)\right), \xi^{\ell}-\alpha^{\ell}(t)\right)_{L^{2}\left(\Omega^{\ell}\right)}, \\
& \quad \xi \in K \text { a.e } t \in[0, T],
\end{aligned}
$$$$
\dot{\beta}(t)=H_{a d}\left(\beta(t), \zeta_{\beta}, R\left(\left|\mathbf{u}_{\tau}^{1}(t)-\mathbf{u}_{\tau}^{2}(t)\right|\right)\right),
$$$$
\mathbf{u}(0)=\mathbf{u}_{0}, \quad \beta(0)=\beta_{0}, \quad \alpha(0)=\alpha_{0} .
$$

We notice that the variational problem PV is formulated in terms of displacement field, damage field and bonding field. The existence of the unique solution of problem PV is stated and proved in the next section. To this end, we consider the following remark which is used in different places of the paper.

Remark 3.2 We note that, in Problem $\mathrm{P}$ and in Problem PV, we do not need to impose explicitly the restriction $0 \leq \beta \leq 1$. Indeed, equation (43) guarantees that $\beta(x, t) \leq \beta_{0}(x)$ and, therefore, assumption (30) shows that $\beta(x, t) \leq 1$ for $t \geq 0$, a.e. $x \in \Gamma_{3}$. On the other hand, if $\beta\left(x, t_{0}\right)=0$ at time $t_{0}$, then it follows from (43) that $\dot{\beta}(x, t)=0$ for all $t \geq t_{0}$ and therefore, $\beta(x, t)=0$ for all $t \geq t_{0}$, a.e. $x \in \Gamma_{3}$. We conclude that $0 \leq \beta(x, t) \leq 1$ for all $t \in[0, T]$, a.e. $x \in \Gamma_{3}$.

\section{An existence and uniqueness result}

Now, we propose our existence and uniqueness result.

Theorem 4.1 Assume that (23)-(32) hold. Then there exists a unique solution of Problem PV. Moreover, the solution satisfies

$$
\begin{gathered}
\mathbf{u} \in C^{1}(0, T ; \boldsymbol{V}), \\
\alpha \in H^{1}\left(0, T ; E_{0}\right) \cap L^{2}\left(0, T ; E_{1}\right), \\
\beta \in W^{1, \infty}\left(0, T ; L^{2}\left(\Gamma_{3}\right)\right) \cap \mathcal{Z} .
\end{gathered}
$$

The functions $\mathbf{u}, \alpha$ and $\beta$ which satisfy (5) and (41)-(44) are called a weak solution of the contact problem $\mathrm{P}$. We conclude that, under the assumptions (23) - (32), the mechanical problem (5)-(15) has a unique weak solution satisfying 
(45)-(47). The regularity of the weak solution is given by (45)-(47) and, in term of stresses,

$$
\boldsymbol{\sigma} \in C\left(0, T ; \mathcal{H}_{1}\right)
$$

Indeed, it follows from (41) that $\operatorname{Div} \boldsymbol{\sigma}^{\ell}+f_{0}^{\ell}=\mathbf{0}$ for all $t \in[0, T]$ and therefore the regularity (45) of $\mathbf{u}$, combined with (23)- (32) implies (48).

The proof of Theorem 4.1 is carried out in several steps that we prove in what follows, everywhere in this section we suppose that assumptions of Theorem 4.1 hold, and we consider that $C$ is a generic positive constant which depends on $\Omega^{\ell}, \Gamma_{1}^{\ell}, \Gamma_{2}^{\ell}, \Gamma_{3}, p_{\tau}, \mathcal{A}^{\ell}, \mathcal{G}^{\ell}, \mathcal{F}^{\ell}, \phi^{\ell}, \kappa^{\ell}$, and $L$. Let a $\eta=\left(\eta^{1}, \eta^{2}\right) \in C(0, T ; \boldsymbol{V})$ be given. In the first step we consider the following variational problem.

\section{Problem PV ${ }_{\eta}^{u}$} that

Find a displacement field $\mathbf{u}_{\eta}=\left(\mathbf{u}_{\eta}^{1}, \mathbf{u}_{\eta}^{2}\right):[0, T] \rightarrow \boldsymbol{V}$ such

$$
\begin{aligned}
& \sum_{\ell=1}^{2}\left(\mathcal{A}^{\ell} \boldsymbol{\varepsilon}\left(\dot{\mathbf{u}}_{\eta}^{\ell}(t)\right), \varepsilon\left(\mathbf{v}^{\ell}\right)\right)_{\mathcal{H}^{\ell}}+(\eta(t), \mathbf{v})_{\boldsymbol{V}}=(\mathbf{f}(t), \mathbf{v})_{\boldsymbol{V}} \\
& \quad \forall \mathbf{v} \in \boldsymbol{V}, t \in(0, T), \\
& \mathbf{u}_{\eta}(0)=\mathbf{u}_{0} .
\end{aligned}
$$

We have the following result for the problem $\mathrm{PV}_{\eta}^{u}$.

Lemma 4.2 There exists a unique solution to problem $P V_{\eta}$ which satisfies the regularity (45).

Proof. We use the Riesz representation theorem to define the operators $A: \boldsymbol{V} \rightarrow \boldsymbol{V}$, such that

$$
(A \mathbf{u}, \mathbf{v})_{\boldsymbol{V}}=\sum_{\ell=1}^{2}\left(\mathcal{A}^{\ell} \varepsilon\left(\mathbf{u}^{\ell}\right), \boldsymbol{\varepsilon}\left(\mathbf{v}^{\ell}\right)\right)_{\mathcal{H}^{\ell}}, \quad \forall \mathbf{u}, \mathbf{v} \in \boldsymbol{V} .
$$

It follows from (51) and (23)(a) that

$$
\|A \mathbf{u}-A \mathbf{v}\|_{\boldsymbol{V}} \leq L_{A}\|\mathbf{u}-\mathbf{v}\|_{\boldsymbol{V}}, \quad \forall \mathbf{u}, \mathbf{v} \in \boldsymbol{V},
$$

which shows that $A: \boldsymbol{V} \rightarrow \boldsymbol{V}$ is Lipschitz continuous. Now, by (51) and (23) (b), we find

$$
(A \mathbf{u}-A \mathbf{v}, \mathbf{u}-\mathbf{v})_{\boldsymbol{V}} \geq m_{A}\|\mathbf{u}-\mathbf{v}\|_{\boldsymbol{V}}^{2}, \forall \mathbf{u}, \mathbf{v} \in \boldsymbol{V},
$$

i.e., that $A: \boldsymbol{V} \rightarrow \boldsymbol{V}$ is a strongly monotone operator on $\boldsymbol{V}$. therefore $A$ is invertible and its inverse $A^{-1}$ is also strongly monotone operator Lipschitz continuous on $\boldsymbol{V}$. Moreover using Riesz Representation Theorem we may define an element $f_{\eta} \in(0, T ; \boldsymbol{V})$ by

$$
\left(\mathbf{f}_{\eta}, \mathbf{v}\right)_{\boldsymbol{V}}=(\mathbf{f}, \mathbf{v})_{\boldsymbol{V}}-(\eta, \mathbf{v})_{\boldsymbol{V}}
$$

It follows now from classical result that there exists a unique function $\mathbf{v}_{\eta} \in(0, T ; \boldsymbol{V})$ which satisfies

$$
A \mathbf{v}_{\eta}(t)=\mathbf{f}_{\eta}(t) .
$$

Let $\mathbf{u}_{\eta}:[0, T] \rightarrow \boldsymbol{V}$ be the function defined by

$$
\mathbf{u}_{\eta}(t)=\int_{0}^{t} \mathbf{v}_{\eta}(s)+\mathbf{u}_{0}, \quad \forall t \in[0, T] .
$$

It follows from (51)-(55) that $\mathbf{u}_{\eta}$ is a solution of the variational problem $P V_{\eta}$ and it satisfies the regularity expressed in (45). This concludes the existence part of lemma1. The uniqueness of the solution follows from the uniqueness of the solution of the problem (54).

In the second step, we use the displacement field $\mathbf{u}_{\eta}$ obtained in Lemma 4.2 and we consider the following initial-value problem.

\section{Problem PV $\mathbf{P V}_{\eta}^{\beta}$}

Find the adhesion $\beta_{\eta}:[0, T] \rightarrow L^{\infty}\left(\Gamma_{3}\right)$ such that

$$
\begin{aligned}
& \dot{\beta}_{\eta}(t)=H_{a d}\left(\beta_{\eta}(t), \zeta_{\beta_{\eta}}(t), R\left(\left|\mathbf{u}_{\tau}^{1}(t)-\mathbf{u}_{\tau}^{2}(t)\right|\right)\right), \\
& \quad \text { a.e. } t \in(0, T), \\
& \beta_{\eta}(0)=\beta_{0} .
\end{aligned}
$$

We have the following result.

Lemma 4.3 There exists a unique solution $\beta_{\eta} \in$ $W^{1, \infty}\left(0, T ; L^{\infty}\left(\Gamma_{3}\right)\right) \cap \mathcal{Z}$ to Problem $\mathrm{PV}_{\eta}^{\beta}$.

Proof. For simplicity we suppress the dependence of various functions on $\Gamma_{3}$, and note that the equalities and inequalities below are valid a.e. on $\Gamma_{3}$. Let $\zeta \in L^{\infty}\left(0, T ; L^{\infty}\left(\Gamma_{3}\right)\right)$ and consider the mapping $H_{\eta \zeta}(t, \cdot): L^{\infty}\left(\Gamma_{3}\right) \rightarrow L^{\infty}\left(\Gamma_{3}\right)$ defined a.a on $[0, T]$ by

$$
H_{\eta \zeta}(t, \beta)=H_{a d}\left(\beta(t), \zeta(t), R\left(\left|\mathbf{u}_{\eta \tau}(t)\right|\right),\right.
$$

The operator $H_{\eta \zeta}$ is continuous and Lipschitz relative to the second argument. as $\beta_{0} \in L^{\infty}\left(\Gamma_{3}\right), t \mapsto H_{\eta \zeta}(t, \beta)$ belongs to $L^{\infty}\left(0, T ; L^{\infty}\left(\Gamma_{3}\right)\right)$. we can apply the Cauchy-Lipschitz theorem and the proof of this theorem see ([24] page 60) which gives us the existence and uniqueness of a function $\beta_{\eta \zeta} \in W^{1, \infty}\left(0, T ; L^{\infty}\left(\Gamma_{3}\right)\right.$ such that

$$
\begin{aligned}
& \dot{\beta}_{\eta \zeta}(t)=H_{a d}\left(\beta_{\eta \zeta}(t), \zeta(t), R\left(\left|\mathbf{u}_{\eta \tau}(t)\right|\right)\right), \\
& \text { a.e. } t \in(0, T), \\
& \beta_{\eta \zeta}(0)=\beta_{0} .
\end{aligned}
$$

It proves that $\beta_{\eta \zeta} \in \mathcal{Z}$. We suppose that $\beta_{\eta \zeta}\left(t_{0}\right)<0$ for some $t_{0} \in[0, T]$. Under the condition (30) we have $0 \leq$ $\beta_{\eta \zeta}(0) \leq 1$ and therefore $t_{0}>0$ moreover, since the mapping $t \mapsto \beta(t):[0, T] \rightarrow \mathbb{R}$ is continuous, we can find $t_{1} \in\left[0, t_{0}\right)$ such that $\beta_{\eta \zeta}\left(t_{1}\right)=0$.

Now, let $t_{2}=\sup \left\{t \in\left[t_{1}, t_{0}\right], \quad \beta_{\eta \zeta}(t)=0\right\}$ then $t_{2}<$ $t_{0}, \quad \beta_{\eta \zeta}\left(t_{2}\right)=0$ and $\beta_{\eta \zeta}(t)<0$ for $t \in\left(t_{2}, t_{0}\right]$. Assumption (28)(e) and equation (58) imply that $\dot{\beta}_{\eta}(t) \geq 0$ for $t \in\left(t_{2}, t_{0}\right]$, and therefore $\beta_{\eta}\left(t_{0}\right) \geq \beta_{\eta}\left(t_{2}\right)=0$ which is a contradiction. We conclude that $\beta_{\eta}(t) \geq 0$ for all $t \in[0, T]$.

A similar argument shows that $\beta_{\eta \zeta}(t) \leq 1$ for all $t \in[0, T]$.

$$
0 \leq \beta_{\eta \zeta}(t) \leq 1, \quad \forall t \in[0, T], \quad \text { a.e. on } \Gamma_{3} .
$$

The property $\beta_{\eta \zeta}(t) \in \mathcal{Z}$ so we can consider the operator $\Lambda_{\eta}: L^{\infty}\left(0, T ; L^{\infty}\left(\Gamma_{3}\right) \rightarrow L^{\infty}\left(0, T ; L^{\infty}\left(\Gamma_{3}\right)\right.\right.$ defined by

$$
\Lambda_{\eta} \zeta(t)=\int_{0}^{t} \beta_{\eta \zeta}(s) d s \quad t \in[0, T]
$$


for which we prove that a single fixed point.

Let $\zeta_{1}, \zeta_{2} \in L^{\infty}\left(0, T ; L^{\infty}\left(\Gamma_{3}\right)\right)$ and let $s \in[0, T]$. From (58),(59) for $i=1,2$ we

$$
\beta_{\eta \zeta_{i}}(s)=\beta_{0}+\int_{0}^{s} H_{a d}\left(\beta_{\eta \zeta_{i}}(\theta), \zeta_{i}(\theta), R\left(\left|\mathbf{u}_{\eta \tau}(\theta)\right|\right)\right) d \theta,
$$

and, using the last equality and (28)(a) we find

$$
\begin{array}{r}
\left|\beta_{\eta \zeta_{1}}(s)-\beta_{\eta \zeta_{2}}(s)\right| \leq L_{a d}\left(\int_{0}^{s}\left|\beta_{\eta \zeta_{1}}(\theta)-\beta_{\eta \zeta_{2}}(\theta)\right| d \theta+\right. \\
\left.\int_{0}^{s}\left|\zeta_{1}(\theta)-\zeta_{2}(\theta)\right| d \theta\right) .
\end{array}
$$

Here and in the following, $C$ is a positive constant which depends on the data but is independent of time and initial conditions, and which may change from line to line.

Applying the Gronwall lemma we find

$$
\left|\beta_{\eta \zeta_{1}}(s)-\beta_{\eta \zeta_{2}}(s)\right| \leq C \int_{0}^{s}\left|\zeta_{1}(\theta)-\zeta_{2}(\theta)\right| d \theta,
$$

and by integrating this inequality in $\Gamma_{3}$ was

$$
\left\|\beta_{\eta \zeta_{1}}(s)-\beta_{\eta \zeta_{2}}(s)\right\|_{L^{\infty}\left(\Gamma_{3}\right)} \leq C \int_{0}^{s}\left\|\zeta_{1}(\theta)-\zeta_{2}(\theta)\right\|_{L^{\infty}\left(\Gamma_{3}\right)} d \theta .
$$

From (61) and (62) we find

$$
\begin{aligned}
& \left\|\Lambda_{\eta} \zeta_{1}(t)-\Lambda_{\eta} \zeta_{2}(t)\right\|_{L^{\infty}\left(\Gamma_{3}\right)} \leq C \\
& \quad \int_{0}^{t} \int_{0}^{s}\left\|\zeta_{1}(\theta)-\zeta_{2}(\theta)\right\|_{L^{\infty}\left(\Gamma_{3}\right)} d \theta d s, \quad t \in[0, T] .
\end{aligned}
$$

Reiterating this inequality $n$ times it comes

$$
\begin{aligned}
& \left\|\Lambda_{\eta}^{n} \zeta_{1}-\Lambda_{\eta}^{n} \zeta_{2}\right\|_{L^{\infty}\left(0, T ; L^{\infty}\left(\Gamma_{3}\right)\right)} \leq \\
& \frac{(C T)^{2 n}}{(2 n) !}\left\|\zeta_{1}-\zeta_{2}\right\|_{L^{\infty}\left(0, T ; L^{\infty}\left(\Gamma_{3}\right)\right)} .
\end{aligned}
$$

It concludes that $n$ sufficiently large, an iterated $\Lambda_{\eta}^{n}$ of $\Lambda_{\eta}$ is a contraction in the Banach space $L^{\infty}\left(0, T ; L^{\infty}\left(\Gamma_{3}\right)\right)$. Then, there exists a unique $\zeta_{\eta}^{*}: \in L^{\infty}\left(0, T ; L^{\infty}\left(\Gamma_{3}\right)\right)$ such that $\Lambda_{\eta}^{n} \zeta_{\eta}^{*}=\zeta_{\eta}^{*}$ and moreover $\zeta_{\eta}^{*}$ is also the unique fixed point $\Lambda_{\eta}$.

Let $\beta_{\eta}=\beta_{\eta \zeta_{\eta}^{*}}$ the solution of (58), (59) for $\zeta=\zeta_{\eta}^{*}$. Using (61) and the relation (34) we obtain

$$
\zeta_{\eta}^{*}(t)=\Lambda_{\eta} \zeta_{\eta}^{*}(t)=\int_{0}^{t} \beta_{\eta} \zeta_{\eta}^{*}(s) d s=\int_{0}^{t} \beta_{\eta}(s) d s=\zeta_{\beta_{\eta}}(t),
$$

$\forall t \in[0, T]$ and keeping in mind (58)-(60) it follows that $\beta_{\eta}$ is a solution to the problem $P_{V}^{\beta}$ and satisfies (47) and $\beta_{\eta} \in$ $\mathcal{Z}$. Which concludes the existence part of Lemma 4.3. The uniqueness follows from the uniqueness of the fixed point of the operator $\Lambda_{\eta}$ given by (61).

In the third step we let $\theta \in C\left(0, T ; E_{0}\right)$ be given and consider the following variational problem for the damage.

\section{Problem $\mathbf{P V}_{\theta}^{\alpha}$}

Find a damage $\alpha_{\theta}=\left(\alpha_{\theta}^{1}, \alpha_{\theta}^{2}\right):[0, T] \rightarrow E$ such that $\alpha_{\theta}(t) \in K$ and

$$
\begin{aligned}
& \sum_{\ell=1}^{2}\left(\dot{\alpha}_{\theta}^{\ell}(t), \xi^{\ell}-\alpha_{\theta}^{\ell}(t)\right)_{L^{2}\left(\Omega^{\ell}\right)}+a\left(\alpha_{\theta}(t), \xi-\alpha_{\theta}(t)\right) \\
& \geq \sum_{\ell=1}^{2}\left(\theta^{\ell}(t), \xi^{\ell}-\alpha_{\theta}^{\ell}(t)\right)_{L^{2}\left(\Omega^{\ell}\right)}, \quad \forall \xi \in K, \\
& \quad \text { a.e. } t \in(0, T),
\end{aligned}
$$

where $K=K^{1} \times K^{2}$. The following abstract result for parabolic variational inequalities (see, e.g., [16, p.47]).

Theorem 4.4 Let $X \subset Y=Y^{\prime} \subset X^{\prime}$ be a Gelfand triple. Let $F$ be a nonempty, closed, and convex set of $X$. Assume that $a(\cdot, \cdot): X \times X \rightarrow \mathbb{R}$ is a continuous and symmetric bilinear form such that for some constants $\alpha>0$ and $c_{0}$,

$$
a(v, v)+c_{0}\|v\|_{Y}^{2} \geq \alpha\|v\|_{X}^{2} \quad \forall v \in X .
$$

Then, for every $u_{0} \in F$ and $f \in L^{2}(0, T ; Y)$, there exists a unique function $u \in H^{1}(0, T ; Y) \cap L^{2}(0, T ; X)$ such that $u(0)=u_{0}, u(t) \in F$ for all $t \in[0, T]$, and

$$
\begin{gathered}
(\dot{u}(t), v-u(t))_{X^{\prime} \times X}+a(u(t), v-u(t)) \geq(f(t), v-u(t))_{Y} \\
\forall v \in F \text { a.e. } t \in(0, T) .
\end{gathered}
$$

We prove next the unique solvability of Problem $\mathrm{PV}_{\theta}^{\zeta}$.

Lemma 4.5 There exists a unique solution $\zeta_{\theta}$ of Problem $P V_{\theta}^{\zeta}$ and it satisfies

$$
\alpha_{\theta} \in H^{1}\left(0, T ; E_{0}\right) \cap L^{2}\left(0, T ; E_{1}\right) .
$$

Proof. The inclusion mapping of $\left(E_{1},\|\cdot\|_{E_{1}}\right)$ into $\left(E_{0}, \| \cdot\right.$ $\left.\| E_{0}\right)$ is continuous and its range is dense. We denote by $E_{1}^{\prime}$ the dual space of $E_{1}$ and, identifying the dual of $E_{0}$ with itself, we can write the Gelfand triple

$$
E_{1} \subset E_{0}=E_{0}^{\prime} \subset E_{1}^{\prime} .
$$

We use the notation $(\cdot, \cdot)_{E_{1}^{\prime} \times E_{1}}$ to represent the duality pairing between $E^{\prime}$ and $E_{1}$. We have

$$
(\alpha, \xi)_{E_{1}^{\prime} \times E_{1}}=(\alpha, \xi)_{E_{0}} \quad \forall \alpha \in E_{0}, \xi \in E_{1},
$$

and we note that $K$ is a closed convex set in $E_{1}$. Then, using (33), (34) and the fact that $\alpha_{0} \in K$ in (32), it is easy to see that Lemma 4.5 is a straight consequence of Theorem 4.4.

Finally as a consequence of these results and using the properties of the operator $\mathcal{G}^{\ell}$, the operator $\mathcal{F}^{\ell}$, the functional $j$ and the functional $\phi^{\ell}$, for $t \in[0, T]$, we consider the element

$$
\Lambda(\eta, \theta)(t)=\left(\Lambda^{1}(\eta, \theta)(t), \Lambda^{2}(\eta, \theta)(t)\right) \in \boldsymbol{V} \times E_{0},
$$

defined by the equations

$$
\begin{aligned}
& \left(\Lambda^{1}(\eta, \theta)(t), \mathbf{v}\right)_{\boldsymbol{V}}=\sum_{\ell=1}^{2}\left(\mathcal{G}^{\ell} \varepsilon\left(\mathbf{u}_{\eta}^{\ell}(t)\right), \varepsilon\left(\mathbf{v}^{\ell}\right)\right)_{\mathcal{H}^{\ell}}+ \\
& j\left(\beta_{\eta}(t), \mathbf{u}_{\eta}(t), \mathbf{v}\right)+ \\
& \sum_{\ell=1}^{2}\left(\int_{0}^{t} \mathcal{F}^{\ell}\left(t-s, \varepsilon\left(\mathbf{u}_{\eta}^{\ell}(s)\right), \alpha_{\theta}^{\ell}(s)\right) d s, \varepsilon\left(\mathbf{v}^{\ell}\right)\right)_{\mathcal{H}^{\ell}} \\
& \forall \mathbf{v} \in \boldsymbol{V},
\end{aligned}
$$


68 Bilateral Contact Problem with Adhesion between Two Bodies for Viscoelastic with Long-term Memory and Damage

$$
\Lambda^{2}(\eta, \theta)(t)=\left(\phi^{1}\left(\varepsilon\left(\mathbf{u}_{\eta}^{1}(t)\right), \alpha_{\theta}^{1}(t)\right), \phi^{2}\left(\varepsilon\left(\mathbf{u}_{\eta}^{2}(t)\right), \alpha_{\theta}^{2}(t)\right)\right)
$$

Here, for every $(\eta, \theta) \in C\left(0, T ; \boldsymbol{V} \times E_{0}\right), \mathbf{u}_{\eta}, \beta_{\eta}$ and $\alpha_{\theta}$ represent the displacement field, bonding field and the damage field obtained in Lemmas 4.2, 4.3 and 4.5 respectively,

We have the following result.

Lemma 4.6 There exists a unique $\left(\eta^{*}, \theta^{*}\right) \in C\left(0, T ; \boldsymbol{V} \times E_{0}\right)$ such that $\Lambda\left(\eta^{*}, \theta^{*}\right)=\left(\eta^{*}, \theta^{*}\right)$.

Proof. Let $\left(\eta_{1}, \theta_{1}\right),\left(\eta_{2}, \theta_{2}\right) \in C\left(0, T ; \boldsymbol{V} \times E_{0}\right)$ and denote by $\mathbf{u}_{i}, \beta_{i}$ and $\alpha_{i}$ the functions obtained in Lemmas 4.2, 4.3, and 4.5 respectively, for $(\eta, \theta)=\left(\eta_{i}, \theta_{i}\right), i=1,2$. Let $t \in$ $[0, T]$. We use (24), (25), (26) and (27) and the definition of $R$, we have

$$
\begin{aligned}
\| \Lambda^{1}\left(\eta_{1}, \theta_{1}\right)(t)- & \Lambda^{1}\left(\eta_{2}, \theta_{1}\right)(t) \|_{\boldsymbol{V}}^{2} \leq \\
& \sum_{\ell=1}^{2}\left\|\mathcal{G}^{\ell} \varepsilon\left(\mathbf{u}_{1}^{\ell}(t)\right)-\mathcal{G}^{\ell} \varepsilon\left(\mathbf{u}_{2}^{\ell}(t)\right)\right\|_{\mathcal{H}^{\ell}}^{2}+ \\
& +\sum_{\ell=1}^{2} \int_{0}^{t} \| \mathcal{F}^{\ell}\left(t-s, \varepsilon\left(\mathbf{u}_{1}^{\ell}(s)\right), \alpha_{1}^{\ell}(s)\right) \\
& -\mathcal{F}^{\ell}\left(t-s, \varepsilon\left(\mathbf{u}_{2}^{\ell}(s)\right), \alpha_{2}^{\ell}(s)\right) \|_{\mathcal{H}^{\ell}}^{2} d s \\
& +\left\|j\left(\beta_{1}(t), \mathbf{u}_{1}(t), \mathbf{v}\right)-j\left(\beta_{2}(t), \mathbf{u}_{2}(t), \mathbf{v}\right)\right\| .
\end{aligned}
$$

Therefore,

$$
\begin{aligned}
& \left\|\Lambda^{1}\left(\eta_{1}, \theta_{1}\right)(t)-\Lambda^{1}\left(\eta_{2}, \theta_{1}\right)(t)\right\|_{\boldsymbol{V}}^{2} \leq C\left(\left\|\mathbf{u}_{1}(t)-\mathbf{u}_{2}(t)\right\|_{V}^{2}\right. \\
& \left.\left.+\int_{0}^{t} \| \mathbf{u}_{1}(s)-\mathbf{u}_{2}(s)\right)\left\|_{\boldsymbol{V}}^{2} d s+\int_{0}^{t}\right\| \alpha_{1}(s)-\alpha_{2}(s)\right) \|_{E_{0}}^{2} d s \\
& \left.\quad+\left\|\beta_{1}(t)-\beta_{2}(t)\right\|_{L^{2}\left(\Gamma_{3}\right)}^{2}\right) .
\end{aligned}
$$

By similar arguments, from (68) and (26) it follows that

$$
\begin{aligned}
& \left\|\Lambda^{2}\left(\eta_{1}, \theta_{1}\right)(t)-\Lambda^{2}\left(\eta_{2}, \theta_{1}\right)(t)\right\|_{E_{0}}^{2} \leq \\
& C\left(\left\|\mathbf{u}_{1}(t)-\mathbf{u}_{2}(t)\right\|_{\boldsymbol{V}}^{2}+\left\|\alpha_{1}(t)-\alpha_{2}(t)\right\|_{E_{0}}^{2}\right) .
\end{aligned}
$$

It follows now from (69) and (70) that

$$
\begin{aligned}
& \left\|\Lambda\left(\eta_{1}, \theta_{1}\right)(t)-\Lambda\left(\eta_{2}, \theta_{1}\right)(t)\right\|_{\boldsymbol{V} \times E_{0}}^{2} \leq C\left(\left\|\mathbf{u}_{1}(t)-\mathbf{u}_{2}(t)\right\|_{\boldsymbol{V}}^{2}\right. \\
& \left.\left.+\int_{0}^{t} \| \mathbf{u}_{1}(s)-\mathbf{u}_{2}(s)\right)\left\|_{\boldsymbol{V}}^{2} d s+\right\| \alpha_{1}(t)-\alpha_{2}(t)\right) \|_{E_{0}}^{2} \\
& \left.\left.\quad+\int_{0}^{t} \| \alpha_{1}(s)-\alpha_{2}(s)\right)\left\|_{E_{0}}^{2} d s+\right\| \beta_{1}(t)-\beta_{2}(t) \|_{L^{2}\left(\Gamma_{3}\right)}^{2}\right) .
\end{aligned}
$$

Also, since

$$
\mathbf{u}_{i}^{\ell}(t)=\int_{0}^{t} \mathbf{v}_{i}^{\ell}(s) d s+\mathbf{u}_{0}^{\ell}(t), \quad t \in[0, T], \ell=1,2,
$$

we have

$$
\left.\left\|\mathbf{u}_{1}(t)-\mathbf{u}_{2}(t)\right\|_{\boldsymbol{V}} \leq \int_{0}^{t} \| \mathbf{v}_{1}(s)-\mathbf{v}_{2}(s)\right) \|_{\boldsymbol{V}} d s .
$$

Moreover, from (49) we obtain that

$$
\begin{aligned}
& \sum_{\ell=1}^{2}\left(\mathcal{A}^{\ell} \varepsilon\left(\mathbf{v}_{1}^{\ell}(t)\right)-\mathcal{A}^{\ell} \boldsymbol{\varepsilon}\left(\mathbf{v}_{2}^{\ell}(t)\right), \varepsilon\left(\mathbf{v}_{1}^{\ell}-\mathbf{v}_{2}^{\ell}\right)\right)_{\mathcal{H}^{\ell}}+ \\
& \left(\eta_{1}-\eta_{2}, \mathbf{v}_{1}-\mathbf{v}_{2}\right)_{\boldsymbol{V}}=0 .
\end{aligned}
$$

We use the assumption (23) and condition (21) to find that

$$
\left.\left\|\mathbf{v}_{1}(t)-\mathbf{v}_{2}(t)\right\|_{\boldsymbol{V}} \leq C \| \eta_{1}(t)-\eta_{2}(t)\right) \|_{\boldsymbol{V}}^{2}
$$

and have

$$
\left\|\mathbf{u}_{1}(t)-\mathbf{u}_{2}(t)\right\|_{\boldsymbol{V}} \leq C \int_{0}^{t}\left\|\eta_{1}(s)-\eta_{2}(s)\right\|_{\boldsymbol{V}} d s \quad \forall t \in[0, T] .
$$

On the other hand, from the Cauchy problem adhesion we can write

$$
\beta_{i}(t)=\beta_{0}+\int_{0}^{t} H_{a d}\left(\beta_{i}(s), \zeta_{\beta_{i}}(s), R\left(\mid \mathbf{u}_{i}(s)\right)\right) d s, i=1,2 .
$$

Using (74), (28)(a) and $R$ we obtain

$$
\begin{aligned}
\left|\beta_{1}(t)-\beta_{2}(t)\right| \leq & L_{H a d} \int_{0}^{t}\left|\beta_{1}(s)-\beta_{2}(s)\right| d s \\
& +L_{H a d} \int_{0}^{t}\left|\zeta_{\beta_{1}}(s)-\zeta_{\beta_{2}}(s)\right| d s \\
& +L_{\text {Had }} \int_{0}^{t}\left|\mathbf{u}_{1}(s)-\mathbf{u}_{2}(s)\right| d s .
\end{aligned}
$$

Using (16) we have

$$
\int_{0}^{t}\left|\zeta_{\beta_{1}}(s)-\zeta_{\beta_{2}}(s)\right| d s \leq C \int_{0}^{t}\left|\beta_{1}(s)-\beta_{2}(s)\right| d s .
$$

Now by (74) and (75) and the Gronwall inequality we

$$
\left|\beta_{1}(t)-\beta_{2}(t)\right|^{2} \leq C \int_{0}^{t}\left|\mathbf{u}_{1}(s)-\mathbf{u}_{2}(s)\right|^{2} d s .
$$

Integrating this inequality $\Gamma_{3}$ and use (22) we obtain

$$
\left\|\beta_{1}(t)-\beta_{2}(t)\right\|_{L^{\infty}\left(\Gamma_{3}\right)}^{2} \leq C \int_{0}^{t}\left\|\mathbf{u}_{1}(s)-\mathbf{u}_{2}(s)\right\|_{V}^{2} d s .
$$

From (65) we deduce that

$$
\begin{aligned}
& \left(\dot{\alpha}_{1}-\dot{\alpha}_{2}, \alpha_{1}-\alpha_{2}\right)_{E_{0}}+a\left(\alpha_{1}-\alpha_{2}, \alpha_{1}-\alpha_{2}\right) \\
& \quad \leq\left(\theta_{1}-\theta_{2}, \alpha_{1}-\alpha_{2}\right)_{E_{0}}, \quad \text { a.e. } t \in(0, T) .
\end{aligned}
$$

Integrating the previous inequality with respect to time, using the initial conditions $\alpha_{1}(0)=\alpha_{2}(0)=\alpha_{0}$ and inequality $a\left(\alpha_{1}-\alpha_{2}, \alpha_{1}-\alpha_{2}\right) \geq 0$, we find

$\frac{1}{2}\left\|\alpha_{1}(t)-\alpha_{2}(t)\right\|_{E_{0}}^{2} \leq \int_{0}^{t}\left(\theta_{1}(s)-\theta_{2}(s), \alpha_{1}(s)-\alpha_{2}(s)\right)_{E_{0}} d s$,

which implies that

$$
\begin{aligned}
& \left\|\alpha_{1}(t)-\alpha_{2}(t)\right\|_{E_{0}}^{2} \leq \int_{0}^{t}\left\|\theta_{1}(s)-\theta_{2}(s)\right\|_{E_{0}}^{2} d s \\
& +\int_{0}^{t}\left\|\alpha_{1}(s)-\alpha_{2}(s)\right\|_{E_{0}}^{2} d s .
\end{aligned}
$$


This inequality combined with Gronwall's inequality leads to

$\left\|\alpha_{1}(t)-\alpha_{2}(t)\right\|_{E_{0}}^{2} \leq C \int_{0}^{t}\left\|\theta_{1}(s)-\theta_{2}(s)\right\|_{E_{0}}^{2} d s \quad \forall t \in[0, T]$.

We substitute (73), (76) and (77) in (71) to obtain

$$
\begin{gathered}
\left\|\Lambda\left(\eta_{1}, \theta_{1}\right)(t)-\Lambda\left(\eta_{2}, \theta_{1}\right)(t)\right\|_{\boldsymbol{V} \times E_{0}}^{2} \leq C \\
\int_{0}^{t}\left\|\left(\eta_{1}, \theta_{1}\right)(s)-\left(\eta_{2}, \theta_{1}\right)(s)\right\|_{\boldsymbol{V} \times E_{0}}^{2} d s .
\end{gathered}
$$

Reiterating this inequality $m$ times we obtain

$$
\begin{aligned}
& \left\|\Lambda^{m}\left(\eta_{1}, \theta_{1}\right)-\Lambda^{m}\left(\eta_{2}, \theta_{1}\right)\right\|_{C\left(0, T ; \boldsymbol{V} \times E_{0}\right)}^{2} \leq \\
& \quad \frac{C^{m} T^{m}}{m !}\left\|\left(\eta_{1}, \theta_{1}\right)-\left(\eta_{2}, \theta_{1}\right)\right\|_{C\left(0, T ; \boldsymbol{V} \times E_{0}\right)}^{2} .
\end{aligned}
$$

Thus, for $m$ sufficiently large, the operator $\Lambda^{m}(\cdot, \cdot)$ is a contraction on the Banach space $C\left(0, T ; \boldsymbol{V} \times E_{0}\right)$, and so $\Lambda(\cdot, \cdot)$ has a unique fixed point.

Now, we have all the ingredients to prove Theorem 4.1 . Proof of Existence. Let $\left(\eta^{*}, \theta^{*}\right) \in C\left(0, T ; \boldsymbol{V} \times E_{0}\right)$ be the fixed point of $\Lambda(\cdot, \cdot)$ and denote

$$
\begin{gathered}
\mathbf{u}_{*}=\mathbf{u}_{\eta^{*}}, \quad \alpha_{*}=\alpha_{\theta^{*}}, \quad \beta_{*}=\beta_{\eta^{*}}, \\
\boldsymbol{\sigma}_{*}^{\ell}=\mathcal{A}^{\ell} \varepsilon\left(\dot{\mathbf{u}}_{*}^{\ell}\right)+\mathcal{G}^{\ell} \varepsilon\left(\mathbf{u}_{*}^{\ell}\right) \\
+\int_{0}^{t} \mathcal{F}^{\ell}\left(t-s, \varepsilon\left(\mathbf{u}_{*}^{\ell}(s)\right), \alpha_{*}^{\ell}(s)\right) d s .
\end{gathered}
$$

We prove that the $\left\{\mathbf{u}_{*}, \alpha_{*}, \beta_{*}\right\}$ satisfies (41)-(44) and the regularities (45)-(47).

Indeed, we write (49) for $\eta=\eta^{*}$ and use (78) to find

$$
\sum_{\ell=1}^{2}\left(\mathcal{A}^{\ell} \varepsilon\left(\dot{\mathbf{u}}_{*}^{\ell}\right), \varepsilon\left(\mathbf{v}^{\ell}\right)\right)_{\mathcal{H}^{\ell}}+\left(\eta^{*}(t), \mathbf{v}\right)_{\boldsymbol{V}}=(\mathbf{f}(t), \mathbf{v})_{\boldsymbol{V}}
$$

$\forall \mathbf{v} \in \boldsymbol{V}$, a.e. $t \in[0, T]$. And we write (65) for $\theta=\theta^{*}$ and use (78) to obtain

$$
\begin{aligned}
& \sum_{\ell=1}^{2}\left(\dot{\alpha}_{*}^{\ell}(t), \xi^{\ell}-\alpha_{*}^{\ell}(t)\right)_{L^{2}\left(\Omega^{\ell}\right)}+a\left(\alpha_{*}(t), \xi-\alpha_{*}(t)\right) \\
& \geq \sum_{\ell=1}^{2}\left(\phi^{\ell}\left(\varepsilon\left(\mathbf{u}_{*}^{\ell}(t)\right), \alpha_{*}^{\ell}(t)\right), \xi^{\ell}-\alpha_{*}^{\ell}(t)\right)_{L^{2}\left(\Omega^{\ell}\right)}
\end{aligned}
$$

for all $\xi \in K$, a.e. $t \in(0, T)$.

Equalities $\Lambda^{1}\left(\eta^{*}, \theta^{*}\right)=\eta^{*}$ and $\Lambda^{2}\left(\eta^{*}, \theta^{*}\right)=\theta^{*}$ combined with (67)-(68) show that

$$
\begin{aligned}
& \left(\eta^{*}(t), \mathbf{v}\right)_{\boldsymbol{V}}=\sum_{\ell=1}^{2}\left(\mathcal{G}^{\ell} \varepsilon\left(\mathbf{u}_{*}^{\ell}\right), \varepsilon\left(\mathbf{v}^{\ell}\right)\right)_{\mathcal{H}^{\ell}}+j\left(\beta_{*}(t), \mathbf{u}_{*}(t), \mathbf{v}\right) \\
& \quad+\sum_{\ell=1}^{2}\left(\int_{0}^{t} \mathcal{F}^{\ell}\left(t-s, \varepsilon\left(\mathbf{u}_{*}^{\ell}(s)\right), \alpha_{*}^{\ell}(s)\right) d s, \varepsilon\left(\mathbf{v}^{\ell}\right)\right)_{\mathcal{H}^{\ell}}, \\
& \forall \mathbf{v} \in \boldsymbol{V} \text {, a.e. } t \in(0, T),
\end{aligned}
$$$$
\theta_{*}^{\ell}(t)=\phi^{\ell}\left(\varepsilon\left(\mathbf{u}_{*}^{\ell}(t)\right), \alpha_{*}^{\ell}(t)\right), \quad \text { a.e. } t \in(0, T), \ell=1,2 \text {. }
$$

We now substitute (81) in (83) to obtain

$$
\begin{aligned}
& \sum_{\ell=1}^{2}\left(\mathcal{A}^{\ell} \varepsilon\left(\dot{\mathbf{u}}_{*}^{\ell}\right)(t), \varepsilon\left(\mathbf{v}^{\ell}\right)\right)_{\mathcal{H}^{\ell}}+\sum_{\ell=1}^{2}\left(\mathcal{G}^{\ell} \varepsilon\left(\mathbf{u}_{*}^{\ell}\right)(t), \varepsilon\left(\mathbf{v}^{\ell}\right)\right)_{\mathcal{H}^{\ell}} \\
& +\sum_{\ell=1}^{2}\left(\int_{0}^{t} \mathcal{F}^{\ell}\left(t-s, \varepsilon\left(\mathbf{u}_{*}^{\ell}(s)\right), \alpha_{*}^{\ell}(s)\right) d s, \varepsilon\left(\mathbf{v}^{\ell}\right)\right)_{\mathcal{H}^{\ell}} \\
& +j\left(\beta_{*}(t), \mathbf{u}_{*}(t), \mathbf{v}\right)=(\mathbf{f}(t), \mathbf{v})_{\boldsymbol{V}} \quad \forall \mathbf{v} \in \boldsymbol{V} \text { a.e. } t \in[0, T],
\end{aligned}
$$

and we substitute (82) in (84) to have $\alpha_{*}(t) \in K$ and

$$
\begin{aligned}
& \sum_{\ell=1}^{2}\left(\dot{\alpha}_{*}^{\ell}(t), \xi^{\ell}-\alpha_{*}^{\ell}(t)\right)_{L^{2}\left(\Omega^{\ell}\right)}+a\left(\alpha_{*}(t), \xi-\alpha_{*}(t)\right) \\
& \geq \sum_{\ell=1}^{2}\left(\phi^{\ell}\left(\varepsilon\left(\mathbf{u}_{*}^{\ell}(t)\right), \alpha_{*}^{\ell}(t)\right), \xi^{\ell}-\alpha_{*}^{\ell}(t)\right)_{L^{2}\left(\Omega^{\ell}\right)},
\end{aligned}
$$

for all $\xi \in K$, a.e. $t \in(0, T)$. And we write (56) for $\eta=\eta^{*}$ and use (78) to find

$$
\dot{\beta}_{*}(t)=H_{a d}\left(\beta_{*}, \zeta_{\beta_{*}} R\left(\left|\mathbf{u}_{* \tau}^{1}(t)-\mathbf{u}_{* \tau}^{2}(t)\right|\right),\right.
$$

\section{a.e. $t \in[0, T]$.}

The relations (85), (86) and (87) allow us to conclude that $\left(\mathbf{u}_{*}, \alpha_{*}, \beta_{*}\right)$ satisfies (41)-(43). Next, (44) and the regularity (45)-(47) follow from Lemmas 4.2, 4.3 and 4.5. Since $\mathbf{u}_{*}$ and $\alpha_{*}$ satisfies (45) and (46), respectively, it follows from (80) that

$$
\boldsymbol{\sigma}_{*} \in C(0, T ; \mathcal{H})
$$

For $\ell=1,2$, we choose $\mathbf{v}=\dot{\mathbf{u}} \pm \phi$ in (85), with $\phi=$ $\left(\phi^{1}, \phi^{2}\right), \phi^{\ell} \in D\left(\Omega^{\ell}\right)^{d}$ and $\phi^{3-\ell}=0$, to obtain

$$
\operatorname{Div} \boldsymbol{\sigma}_{*}^{\ell}(t)=-\boldsymbol{f}_{0}^{\ell}(t) \quad \forall t \in[0, T], \quad \ell=1,2,
$$

where $D\left(\Omega^{\ell}\right)$ is the space of infinitely differentiable real functions with a compact support in $\Omega^{\ell}$. We use (29) and (88) to find

$$
\boldsymbol{\sigma}_{*} \in C\left(0, T ; \mathcal{H}_{1}\right)
$$

Finally we conclude that the weak solution $\left\{\mathbf{u}_{*}, \boldsymbol{\sigma}_{*}, \alpha_{*}, \beta_{*}\right\}$ Problem PV has the regularity (45)(48), which concludes the existence part of Theorem 4.1.

Proof of Uniqueness. The uniqueness of the solution is a consequence of the uniqueness of the fixed point of the operator $\Lambda$ defined by (66)-(68) and the unique solvability of the Problems $\mathrm{PV}_{\eta}^{u}, \mathrm{PV}_{\eta}^{\beta}$, and $\mathrm{PV}_{\eta}^{\alpha}$.

\section{REFERENCES}

[1] M.Campo, J.R. Fernandez and A. Rodriguez-Aros; A quasistatic contact problem with normal compliance and damage involving viscoelastic materials with long memory, Applied Numerical Mathematics 58(2008), 1274-1290. 
70 Bilateral Contact Problem with Adhesion between Two Bodies for Viscoelastic with Long-term Memory and Damage

[2] C. Ciulcu, D. Motreanu and M. Sofonea; Analysis of an elastic contact problem with slip dependent coefficient of friction, Mathematical Inequalities \& Applications 4 (2001), 465-479.

[3] M. Frémond, B. Nedjar; Damage in concrete: The unilateral phenomenon, Nucl. Eng. Des. 156 (1995)323-335.

[4] M. Frémond, B. Nedjar; Damage, gradient of damage and principle of virtual work, Int. J. Solids Stuct 33(8) (1996) 1083-1103.

[5] M. Frémond, K. L. Kuttler, and M. Shillor; Existence and uniqueness of solutions for a one-dimensinal damage model, J. Math. Anal. Appl. 229 (1999) 271-294.

[6] I. R. Ionescu and J.C. Paumier; On the contact problem with slip displacement dependent friction in elastostatics, Int. J. Engng. Sci. 34 (1996), 471-491.

[7] N. Kikuchi and J. T. Oden; Contact Problems in Elasticity, A Study of Variational Inequalities and Finite Element Methods, SIAM, Philadelphia, 1988.

[8] K. L. Kuttler and M. Shillor; Existence for models of damage, preprint 2001.

[9] J. A. C. Martins, J. T. Oden; Existence and uniqueness results for dynamic contact problems with nonlinear normal and friction interface laws, Nonlinear Anal. TMA 11 (1987) $407-428$.

[10] D. Motreanu and M. Sofonea; Quasivariational inequalities and applications in frictional contact problems with normal compliance, Adv. Math. Sci. Appl. 10 (2000), 103-118.

[11] J. Nečas, and I. Hlaváček; Mathematical Theory of Elastic and Elastico-Plastic Bodies: An Introduction, Elsevier Scientific Publishing Company, Amsterdam, Oxford, New York, 1981.

[12] J. T. Oden and J. A. C. Martins; Models and computational methods for dynamic friction phenomena, Computer Methods in Applied Mechanics and Engineering 52 (1985), 527-634.

[13] M. Rochdi, M. Shillor and M. Sofonea; A quasistatic viscoelastic contact problem with normal compliance and friction, J. Elasticity 51 (1998) 105-126.
[14] M. Rochdi, M. Shillor and M. Sofonea; Analysis of a quasistatic viscoelastic problem with friction and damage, $A d v$. Math. Sci. Appl. 10 (2002) 173-189.

[15] M.Selmani, L. Selmani; A Dynamic Frictionless Contact Problem with Adhesion and Damage, Bull. Pol. Acad. Sci., Math 55 (2007), 17-34.

[16] M. Sofonea, W. Han and M. Shillor; Analysis and Approximation of Contact Problems with Adhesion or Damage, Pure and Applied Mathematics. 276, Chapman-Hall/CRC Press, New York, 2006.

[17] A. Touzaline; Analysis of a viscoelastic frictionless contact problem with adhesion, Rev. Roum. Math. Pur. Appl. 55 (2010), 411-430.

[18] L. Zhor, Z.Zellagui, H. Benseridi and S. Drabla; Variational analysis of an electro viscoelastic contact problem with friction, J. Assoc. Arab. Univ. Bas. and Appl. (2013), 1-8.

[19] O. Chau, M. Shillor M. Sofonea, Dynamic frictionless contact with adhesion, J. of Appl. Math. and Phys. (ZAMP), 55, (2004), pp.32-pp.47

[20] O. Chau, M. Shillor, M. Sofonea, Dynamic Frictionless Contact with adhesion, J. Appl. Meth. Phys. (ZAMP), 55, (2004), pp.431-pp.465

[21] M. Frmond, Adhrence des solides, J Mcanique Thorique et applique, 6, (1987), pp.383-pp.407

[22] M. FRMOND, Equilibre des structures qui adhrent leur support, C.R. Acad. Sci. Paris, Sr.II.295,(1982), pp.913-pp.916

[23] L. JiAnu, M. Shillor, M.SofoneA, A viscoelastic bilateral frictionless contact problem with, Applic.Anal 80, (2001), pp.233-pp.255

[24] M. Sofonea And A. Matei, An Elastic Contact Problem with Adhesion and Normal Compliance.preprint. 